\title{
Radon Transform Algorithm for Fingerprint Core Point Detection
}

\author{
Miguel Mora-González ${ }^{1}$, Julio C. Martínez-Romo ${ }^{2}$, Jesús Muñoz-Maciel ${ }^{1}$, \\ Guillermo Sánchez-Díaz ${ }^{3}$, Javier Salinas-Luna ${ }^{3}$, H.I. Piza-Dávila ${ }^{4}$, \\ Francisco J. Luna-Rosas ${ }^{2}$, and Carlos A. de Luna-Ortega ${ }^{1}$ \\ ${ }^{1}$ Universidad de Guadalajara (UdeG), Centro Universitario de los Lagos, Av. Enrique \\ Díaz de León 1144, Col. Paseos de la Montaña, C.P. 47460, Lagos de Moreno, Jal., México \\ mmora@culagos.udg.mx, jesnek@hotmail.com, \\ alejandro.deluna@upa.edu.mx \\ ${ }^{2}$ Instituto Tecnológico de Aguascalientes, Av. A. López Mateos 1801 Ote. Col. Bona Gens, \\ C.P. 20256, Aguascalientes, Ags., México \\ jucemaro@yahoo.com, fjluna@ita.mx \\ ${ }^{3}$ UdeG, Centro Universitario de los Valles, Km 45.5 carr. Guadalajara-Ameca, \\ C.P. 46600, Ameca, Jal., México \\ guillermo.sanchez@profesores.valles.udg.mx, \\ javier.salinas@profesores.valles.udg.mx \\ ${ }^{4}$ Instituto Tecnológico y de Estudios Superiores de Occidente, Apdo. Post. 31-175, \\ C.P. 45604, Tlaquepaque, Jal., México \\ hpiza@iteso.mx
}

\begin{abstract}
This article presents an innovative technique for solving the problem of finding the core within a fingerprint. The Radon transform and a tree clustering algorithm were key to locating the coordinates of the core. Binarization and high-pass filtering processes to improve the contrast in fingerprints are proposed. The core of a fingerprint is located in the geometric cross section of maxima and minima in the Radon transforms at $0^{\circ}$ and $90^{\circ}$. The technique is very stable, since it only presents difficulties when the fingerprint core is located on the edges of the image or is nonexistent.
\end{abstract}

Keywords: Core Point, Fingerprint, Edge Detection, Radon Transform.

\section{Introduction}

Fingerprint analysis is a rather common method for identifying people. This is so because of two biometrical features of fingerprints: they do not change with time and they are unique for each person [1]. That is the reason fingerprint recognition has a wide field of application in security and recognition systems.

There are different parameters to identify in a fingerprint: ridges, rows, deltas, cores, etc., the latter being the parameter around which the others converge. Due to its importance, many authors have implemented several techniques to identify the geometrical position of the core within the image of a fingerprint. At first they found the core by dividing the image into sub-images [2], [3]. Then, curvature detection 
methods and region geometry were used [4]. Others began to use the Poincare Index [5], [6], [7]. Finally, some researchers worked with the orientation, segmentation [8] and curvature [9] patterns of the fingerprint.

In this work, we implemented an alternative approach to finding the core in a fingerprint by using the Radon Transform (RT) as a means to quantify the grey levels between the curves generated by ridges and rows of each fingerprint. We also performed an analysis in convolution filters to improve the images in the process for obtaining the RT. Finally, a tree clustering algorithm is used to match the core coordinates through empathy between the horizontal and vertical RTs of a fingerprint. It was decided to use RT as a method to find the core of a fingerprint because its algorithm is easy and quick to implement and because the results are very straightforward.

In the following sections, the proposed methodology to develop the fingerprint core-locating algorithm is presented. Then, the experimental setup for capturing fingerprints is explained. After that, the experimental results obtained through the proposed algorithm are shown and discussed. Finally the conclusions of the results are expressed.

\section{Methodology}

The motivation to use RT as a method to locate the core in fingerprints came up from observing the shape of the prints themselves, since the cores in the great majority of them are shown as a collection of concentric circles. When the grey levels (GL) around the circles are analyzed, it is observed that the highest GL is in the convergence point of such circles.

In order to find the core in the fingerprint, the following digital processes need to be applied to the image: binarization, convolution, Radon transform, least squares, and tree clustering algorithm. These processes are detailed below.

\subsection{Image Binarization}

A large amount of GL is obtained through the scanning of fingerprints. This also depends on the way the image is scanned. Ideally, there should be only one GL binary range, i.e. two GL (0 and 1$)$. To perform the binarization, an intermediate grey level is chosen as a threshold value, thus making the binarized image obtainable through these two equations:

$$
\operatorname{Im}_{b}(x, y)= \begin{cases}\operatorname{Im}(x, y)<G L_{m}, & 0 \\ \operatorname{Im}(x, y)>G L_{m}, & 1\end{cases}
$$

and

$$
\operatorname{Im}_{n}(x, y)=1-\operatorname{Im}_{b}(x, y),
$$

where $\operatorname{Im}, \operatorname{Im}_{b}, \operatorname{Im}_{n},(x, y)$ and $G L_{m}$ are the original image, the binarized image, the negative, the coordinates of each image and the GL threshold, respectively. An unprocessed fingerprint (Im, the location of the core is enclosed) can be observed in 


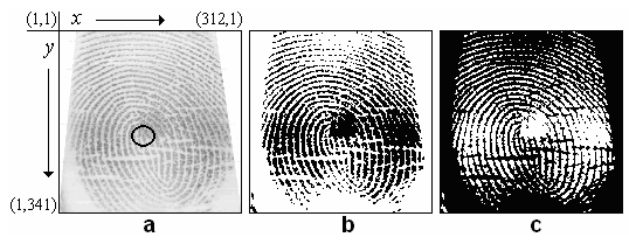

Fig. 1. Fingerprint binarization, a) original fingerprint, b) binary fingerprint, and c) negative fingerprint. Threshold $G L_{m}=200 \mathrm{GL}$.

Fig. 1a; the binarized image $\left(\operatorname{Im}_{b}\right)$ is shown in Fig. $1 \mathrm{~b}$; and the negative $\left(\operatorname{Im}_{n}\right)$ is presented in Fig. 1c.

\subsection{Convolution}

The image of a binarized fingerprint shows the ridges and rows as the skeleton of the original image. However, it is necessary to highlight the data in order to obtain more defined lines and thus be able to get a clearer distinction between ridges and rows (distinguishing the edges). The most common method to detect the edges of an image is spatial filtering, which convolutions the image against a high-pass mask. Convolution mask types that highlight the edges (for North, South, East, and West orientation) are gradient and relief. These types of masks are shown in Table 1 [10], [11]. The mathematical model of discrete convolution applied to images is defined as [12]

$$
\operatorname{Im}(x, y) * h p(x, y)=\sum_{m=-\frac{M+1}{2}}^{\frac{M+1}{2}} \sum_{n=-\frac{N+1}{2}}^{\frac{N+1}{2}} \operatorname{Im}(m, n) h p(x-m, y-n),
$$

where $h p,(m, n)$ and $M x N$ are the convolution mask, the coordinates where the convolution is performed, and the size of the convolution masks, respectively.

Table 1. Some edge-detecting matrices or masks

\begin{tabular}{ccccccccccccccc}
\hline & \multicolumn{3}{c}{ North } & \multicolumn{3}{c}{ South } & \multicolumn{3}{c}{ East } & \multicolumn{3}{c}{ West } \\
\hline \multirow{3}{*}{ Gradient } & 1 & 1 & 1 & -1 & -1 & -1 & -1 & 1 & 1 & & 1 & 1 & -1 \\
& 1 & -2 & 1 & & 1 & -2 & 1 & -1 & -2 & 1 & & 1 & -2 & -1 \\
& -1 & -1 & -1 & 1 & 1 & 1 & -1 & 1 & 1 & & 1 & 1 & -1 \\
\multirow{3}{*}{ Relief } & 1 & 1 & 1 & -1 & -1 & -1 & -1 & 0 & 1 & & 1 & 0 & -1 \\
& 0 & 1 & 0 & 0 & 1 & 0 & -1 & 1 & 1 & & 1 & 1 & -1 \\
& -1 & -1 & -1 & 1 & 1 & 1 & & -1 & 0 & 1 & & 1 & 0 & -1 \\
\hline
\end{tabular}

The masks shown in Table 1 vary on their performance, depending on the characteristics of the fingerprint to be convolutioned. Images in Fig. 2 are the result of the application of equation 3 (through masks in Table 1) to the fingerprint in Fig. 1a. In Fig. 2 we can observe the edges detection being highlighted in four directions. Combinations such as Northeast, Southeast, Northwest and Southwest are also possible. 

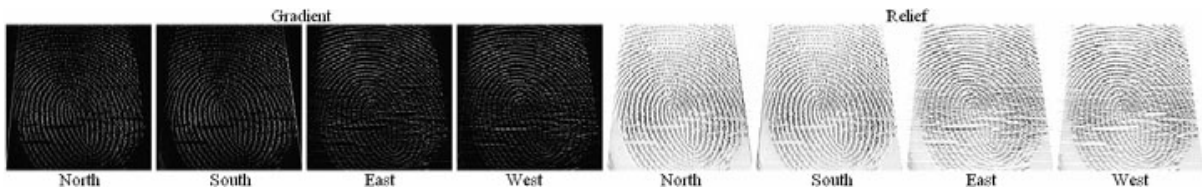

Fig. 2. Skeletonization of a fingerprint (Fig 1a) through Table 1 convolution masks

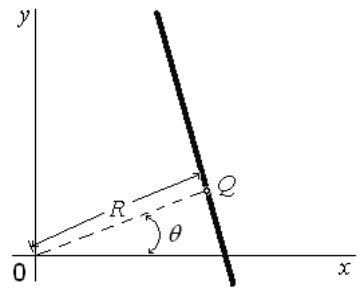

Fig. 3. Radon transform parameters

\subsection{Radon Transform}

The Radon transform may be considered the image's grey levels projection over a given angle with respect to the $x$ axis. The RT mathematical model is [13]

$$
\Re\{\operatorname{Im}(x, y)\}=\int_{-\infty}^{\infty} \int_{-\infty}^{\infty} \operatorname{Im}(x, y) \delta(R-x \cos \theta-y \sin \theta) d x d y,
$$

where $\Re, \delta, R$ and $\theta$ are the Radon transform operator, the unit impulse function, the distance from the origin to the profile line and the angle of direction of the same line, respectively. Each of these parameters can be observed in fig. 3, where $Q$ is the origin of the profile line to be obtained (thick bold line). We find the Radon transform useful in the detection cores within fingerprints due to its ability to detect lines; a fingerprint may be considered roughly as composed of vertical and horizontal lines with the core located at the intersection of such lines. Radon transforms (with $\theta=0^{\circ}$ and $90^{\circ}, \mathrm{RT}_{0}$ and $\mathrm{RT}_{90}$ ) for fingerprints from figures 1 and 2 are shown in Fig. 4 . It can be observed that the core is the point in the fingerprint where $\mathrm{RT}_{0}$ minima and $\mathrm{RT}_{90}$ maxima intersect (dotted lines).

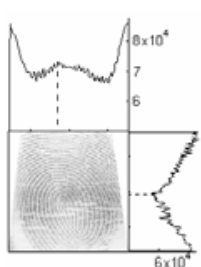

a

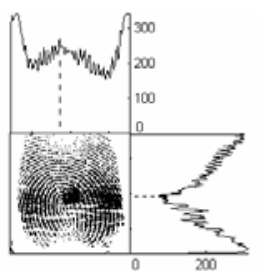

b

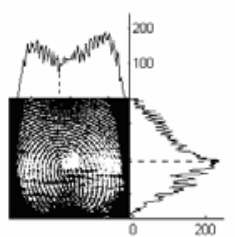

c

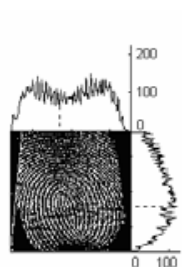

d

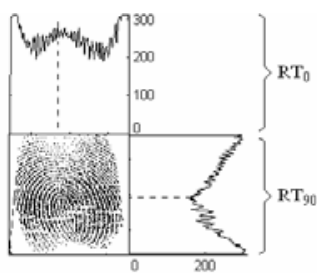

e

Fig. 4. Radon transform $0^{\circ}$ and $90^{\circ}$ for: a) original fingerprint, b) binarized, c) negative, d) north gradient, and e) south relief image. Core point is in the intersection of dotted lines. 


\subsection{Curve fitting by Least Squares}

Due to the need to adjust the RTs for better identification of maxima and minima, least squares method has been employed. The basic idea is fitting the data obtained from RTs through the polynomial

$$
h(x)=\sum_{j=1}^{k+1} c_{j} x^{k-j-1},
$$

calculating the deviation of the curve with respect to the polynomial through [14]

$$
r_{i}=f_{i}-h\left(x_{i}\right) \text {, with } i=1,2, \cdots, I,
$$

where $c, k, f$, and $I$ are the polynomial coefficients, the polynomial degree, the data to be fit, and the number of data, respectively. Thus, the addition of deviations raised to the second power is represented by the following equation

$$
D=\sum_{i=1}^{I} r_{i}^{2},
$$

which is why the minimum of every squared deviation will become evident when the partial derivative with respect to each coefficient $c$ is zero. This is

$$
\frac{\partial D}{\partial c_{j}} D=0,
$$

when substituting equations (5-7) in equation (8) and partially deriving them against each coefficient, we obtain

$$
\sum_{j=1}^{n+1}\left[\sum_{i=1}^{N} x_{i}^{n-j-1+k}\right] \cdot c_{j}=\sum_{i=1}^{N} x_{i}^{k} f_{i} .
$$

Polynomial $h(x)$ coefficients can be obtained through equation (9), which results in the parameters needed for the least squares fit.

\subsection{Tree Clustering Algorithm}

In order to find the minima and maxima observed in the RTs of images shown above, a tree clustering algorithm is proposed. This algorithm performs a comparative adjustment between data and threshold value. It detects sign changes in the data function slope. The algorithm consists of the following steps [15]:

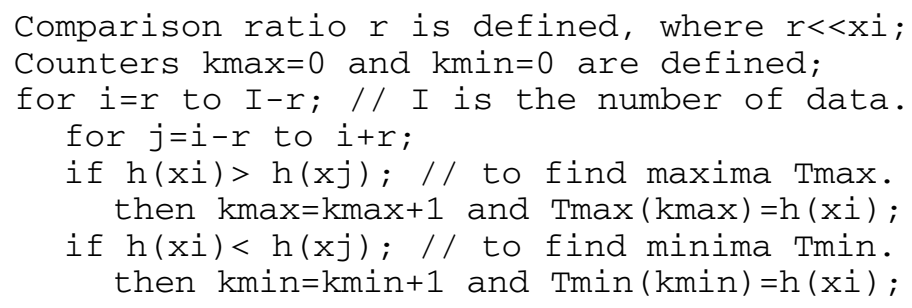


maxima=Tmax(1); // initialization variable maxima.

for $i=1$ to $\mathrm{kmax}-1$; // to find principal maxima.

if $\operatorname{Tmax}(i+1)>\operatorname{Tmax}(i)$; then maxima $=\operatorname{Tmax}(i+1)$;

minima=Tmin(1); // initialization variable minima.

for $j=1$ to kmin; // to find principal minima.

if $\operatorname{Tmin}(j+1)<\operatorname{Tmin}(j)$;

then minima $=\operatorname{Tmin}(j+1)$;

Finding all the maxima and minima in a signal will depend on two factors: signal noise and size of $r$. It is recommended that the noise frequency be lower than $2 r$, thus fitting into Nyquist criterion [16].

\section{Experimental Setup}

The setup used to capture fingerprints is quite simple: a Microsoft FingerPrint scanner connected via USB to a PCG-K35F Sony Vaio laptop. The scanner produces $355 \times 390$-pixel images in BMP format. The images have a margin with no information (electronically removed), resulting in 312×341-pixel images. Matlab® was the software used to implement the methods seen in the previous section.

\section{Experimental Results}

Based on the results from Fig. 4, it can be observed that the core perimeter is signaled by a series of maxima and minima in the RT graphs. The problem with such graphs is the excessive noise they contain, which is generated by the high number of grey levels and/or the low contrast between rows and ridges in each of the sub-images shown in Figure 4. It was also observed that images with a black background (negative and gradient in Fig. 4) had a smaller amount of maxima and minima, which facilitates pinpointing the cores. It was then decided that an adjustment had to be made in the RT graphs using least square fitting. This softens the RTs graphs without losing relevant information in them, as can be seen comparing Figures 4 and 5.

Table 2. Core coordinates and standard deviations for 7 individuals' fingerprints using different convolution and binarization parameters. Where: $\mathrm{A}=$ Binarizated gradient, $\mathrm{B}=$ Binarizated negative relief, $\mathrm{P}=$ Process, $\mathrm{S}=$ Sample and $\mathrm{GL}_{\mathrm{m}}=180$.

\begin{tabular}{cccccccccc}
\hline \multirow{2}{*}{$\mathrm{S}$} & $\mathrm{P}$ & \multicolumn{9}{c}{ Core coordinates and $\sigma$ (in pixels) } \\
& & $\left(x_{n}, y_{n}\right)$ & $\left(x_{s}, y_{s}\right)$ & $\left(x_{e}, y_{e}\right)$ & $\left(x_{w}, y_{w}\right)$ & $\left(x_{c}, y_{c}\right)$ & $\sigma_{\mathrm{x}}$ & $\sigma_{\mathrm{y}}$ & Fig. \\
\hline & $\mathrm{A}$ & $(143,189)$ & $(152,188)$ & $(155,115)$ & $(156,87)$ & $(152,145)$ & 5.9 & 51.8 & $5 \mathrm{c}$ \\
& $\mathrm{B}$ & $(147,186)$ & $(140,186)$ & $(147,180)$ & $(148,188)$ & $(146,185)$ & 3.7 & 3.4 & $5 \mathrm{~d}$ \\
& $\mathrm{~A}$ & $(166,216)$ & $(147,237)$ & $(154,154)$ & $(171,176)$ & $(160,196)$ & 11 & 37.7 & $6 \mathrm{a}$ \\
2 & $\mathrm{~B}$ & $(157,199)$ & $(152,202)$ & $(154,201)$ & $(154,208)$ & $(154,203)$ & 2 & 3.9 & $6 \mathrm{~b}$ \\
3 & $\mathrm{~B}$ & $(138,180)$ & $(131,173)$ & $(168,85)$ & $(167,88)$ & $(151,132)$ & 19.3 & 52.1 & $6 \mathrm{c}$ \\
4 & $\mathrm{~B}$ & $(119,183)$ & $(116,185)$ & $(112,214)$ & $(117,221)$ & $(116,201)$ & 2.9 & 19.6 & $6 \mathrm{~d}$ \\
5 & $\mathrm{~B}$ & $(156,173)$ & $(149,177)$ & $(162,175)$ & $(162,191)$ & $(157,179)$ & 6.2 & 8.2 & $6 \mathrm{e}$ \\
6 & $\mathrm{~B}$ & $(174,200)$ & $(173,209)$ & $(143,77)$ & $(142,77)$ & $(158,141)$ & 18 & 73.7 & $6 \mathrm{f}$ \\
& $\mathrm{A}$ & $(103,317)$ & $(242,316)$ & $(235,261)$ & $(173,226)$ & $(188,280)$ & 64.7 & 44.5 & $6 \mathrm{~g}$ \\
7 & $\mathrm{~B}$ & $(171,243)$ & $(166,202)$ & $(178,243)$ & $(173,255)$ & $(172,236)$ & 5 & 23.2 & $6 \mathrm{~h}$ \\
\hline
\end{tabular}




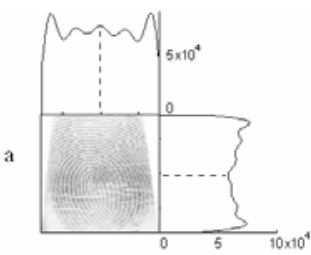

c

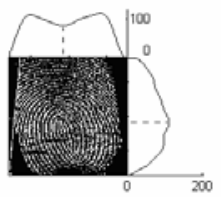

d

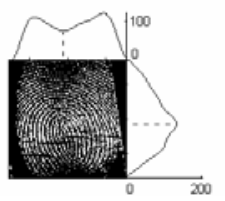

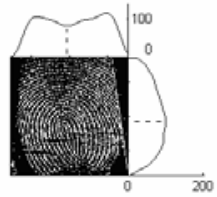

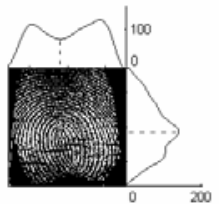

b
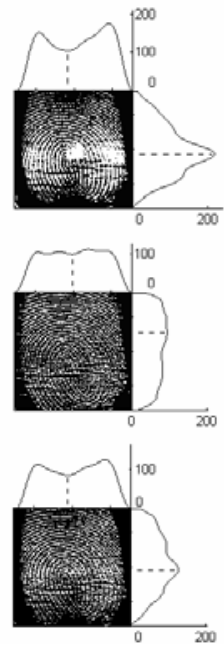
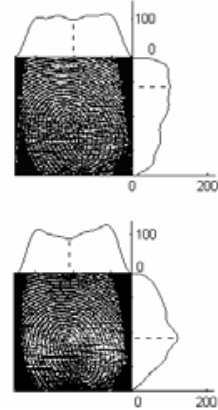

Fig. 5. Softened RTs at $0^{\circ}$ and $90^{\circ}$ for a) original fingerprint individual 1 ; b) binarized negative image; North, South, East, West images for c) binarized gradient; and d) binarized negative relief. $(x, y),\left(x_{n}, y_{n}\right)\left(x_{s}, y_{s}\right),\left(x_{e}, y_{e}\right)$ and $\left(x_{w}, y_{w}\right)$ core points are in the intersection of dotted lines.

We began by comparing the adjusted RT graphs using a 21th grade polynomial, thus it was possible to fit the curves without a lost of maxima or minima of the original RT. The softened RTs from the original fingerprint and the softened RTs for the 4 directions of the binarized gradient and the binarized relief are shown in Fig. 5. A negative relief was used in order to obtain images with a black background. It was observed that images processed with relief-type convolution masks only have one maximum in $\mathrm{TR}_{90}$ and a minimum in $\mathrm{TR}_{0}$, whereas images processed with gradients have, in most cases, several maxima and minima. The reason is that relief-processed images have a better contrast than gradient-processed images. Maxima and minima were obtained through a tree clustering algorithm (described in section 2.5), given the fact that image skeletonization processes (North, South, East, West) both for gradients and reliefs have a slight image deviation in the sense of direction of the process to be carried out. Then, it was decided to obtain the core location using the average of the 4 minima for the $\mathrm{RT}_{0}$ and the 4 maxima for the $\mathrm{RT}_{90}$. This is

$$
x_{c}=\left(x_{n}+x_{s}+x_{e}+x_{w}\right) / 4 \text {, }
$$

and

$$
y_{c}=\left(y_{n}+y_{s}+y_{e}+y_{w}\right) / 4,
$$

where $\left(x_{c}, y_{c}\right),\left(x_{n}, y_{n}\right),\left(x_{s}, y_{s}\right),\left(x_{e}, y_{e}\right)$ and $\left(x_{w}, y_{w}\right)$ are the coordinates of the core in the original, North, South, East, and West images, respectively. The standard deviation was also calculated for coordinates in $\mathrm{x}\left(\sigma_{\mathrm{x}}\right)$ and for coordinates in $\mathrm{y}\left(\sigma_{\mathrm{y}}\right)$. This was done to observe a possible core deviation with respect to the real one. We obtained softened RTs for other 6 individuals' fingerprints, whose core shapes and core positions are different (Fig. 6). Table 2 shows the results of the calculations of core coordinates of the 7 individuals' fingerprints. 


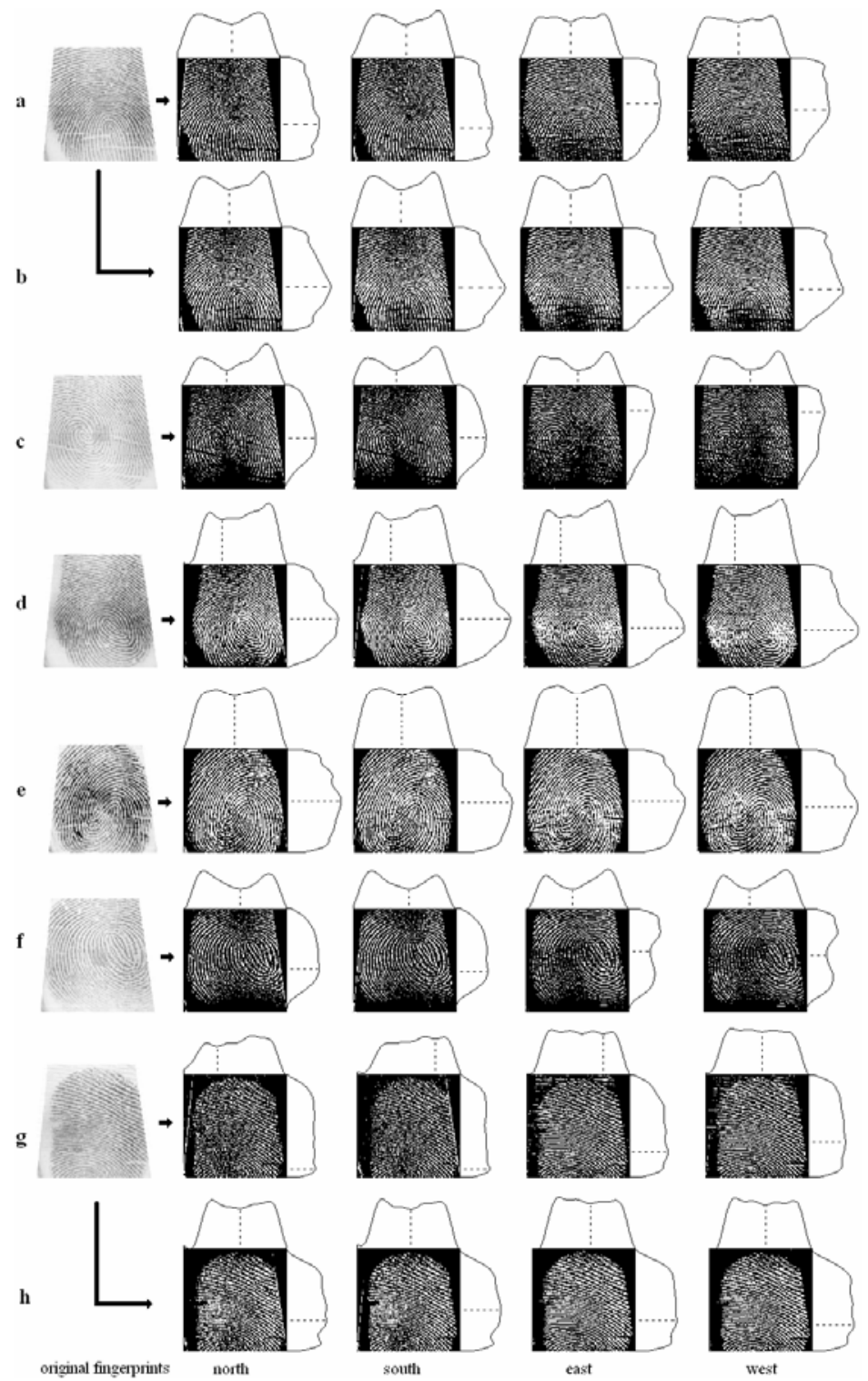

Fig. 6. Softened RTs at $0^{\circ}$ and $90^{\circ}$ for 6 individuals. $\left(x_{n}, y_{n}\right)\left(x_{s}, y_{s}\right),\left(x_{e}, y_{e}\right)$ and $\left(x_{w}, y_{w}\right)$ core points are in the intersection of dotted lines

\section{Conclusions}

A new technique for locating cores in fingerprints has been described. The Radon transform was used as a mathematical tool to locate the cores. RTs are algorithms which are fast to process and to interpret in small images. Therefore it was relatively fast to identify maxima and minima that pointed at the core coordinates. 
The technique described above was very stable when used in one or two core fingerprints. However, it destabilizes when the core is hidden. There are two possible cases: a) when the core is hidden in North or South positions, there is more than one maximum in $\mathrm{RT}_{90}$; b) when the core is hidden in the East or West positions, there is more than one minimum in $\mathrm{RT}_{0}$.

By applying only RT and least square fitting to a fingerprint works fine when dealing with well-scanned or high contrast images. In fingerprints with digitalization mistakes (low contrast or missing data,) however, it is better to apply convolution masks (gradient or relief) before in order to obtain more defined rows and ridges and average the 4 pairs of the softened RTs at the end.

The best convolution mask option depends on the digitalization and type of fingerprint. The results show that masks that presented a higher accuracy finding the core were those in which the standard deviation was minimal, it is to say, when $\sigma_{x} \rightarrow 0$ and $\sigma_{\mathrm{y}} \rightarrow 0$.

The core in a fingerprint is the most important parameter. Consequently, being able to find it correctly is a major step in the identification of people through fingerprint recognition. For double-cored fingerprints, the central point between the two cores was located (see Fig. 6e).

The core was identified localizing the minima of the $\mathrm{RT}_{0}$ and the maxima of the $\mathrm{RT}_{90}$ using a tree clustering algorithm. These minima and maxima are global ones; however the minima of the $\mathrm{RT}_{0}$ should discard the borders since they do not contain the fingerprint information.

Finally, we found that the proposed method, as compared with others, does not require high resolution images (with 312×341-pixel images were enough); the speed of the algorithm is similar to Poincaré and Edge Map methods and showed a high repeatability rate in the detection of single and double core in fingerprints.

Acknowledgments. The authors would like to thank David A. Leith for his contributions to the development of this work. Also Mr. Mora would like to acknowledge to the Centro Universitario de los Lagos (UdeG) for financing his research stay at Instituto Tecnológico de Aguascalientes in the summer of 2008.

\section{References}

1. Cho, B.-H., Kim, J.-S., Bae, J.-H., Bae, I.-G., Yoo, K.-Y.: Core-based Fingerprint Image Classification. In: 15th International Conference on Pattern Recognition, pp. 859-862. IEEE Press, Barcelona (2000)

2. Kameswara Rao, C.V., Balck, K.: Finding the core point in fingerprint. IEEE Trans. Comp. C-27(1), 77-81 (1978)

3. Ohtsuka, T., Kondo, A.: Improvement of the fingerprint core detection using extended relation graph. In: Nonlinear Signal and Image Processing, p. 20. IEEE Press, Sapporo (2005)

4. Julasayvake, A., Choomchuay, S.: An algorithm for fingerprint core point detection. In: 9th International Symposium on Signal Processing and Its Applications, pp. 1-4. IEEE Press, Sharjah (2007)

5. Liu, M., Jiang, X., Kot, A.C.: Fingerprint Referente Point Detection. In: Zhang, D., Jain, A.K. (eds.) ICBA 2004. LNCS, vol. 3072, pp. 272-279. Springer, Heidelberg (2004) 
6. Khan, N.Y., Javed, M.Y., Khattak, N., Chang, U.M.Y.: Optimization of core point detection in fingerprints. In: Digital Image Computing Techniques and Applications, pp. 260 266. IEEE Press, Glenelg (2007)

7. Ohtsuka, T., Watanabe, D., Tomizawa, D., Hasegawa, Y., Aoki, H.: Reliable detection of core and delta in fingerprints by using singular candidate method. In: Computer Vision and Pattern Recognition Workshops, pp. 1-6. IEEE Press, Anchorage (2008)

8. Akram, M.U., Tariq, A., Nasir, S., Khanam, A.: Core point detection using improved segmentation and orientation. In: Computer Systems and Applications, pp. 637-644. IEEE Press, Doha (2008)

9. Sun, Q.-s., Mao, Z., Mei, Y.: Detection of Core Points in Fingerprint Images Based on Edge Map. In: 2009 International Conference on Electronic Computer Technology, pp. 126-129. IEEE Press, Macau (2009)

10. Vélez, J.F., Moreno, F.B., Calle, A.S., Sánchez-Marín, J.L.E.: Visión por computador. Dykinson, Madrid (2003)

11. Bow, S.: Pattern Recognition and Image Preprocessing. Marcel Dekker, New York (2002)

12. Gonzalez, R.C., Woods, R.E.: Digital Image Processing. Prentice-Hall, New Jersey (2002)

13. Bracewell, R.N.: Two-Dimensional Imaging. Prentice Hall, New Jersey (1995)

14. Nakamura, S.: Métodos numéricos aplicados con software. Pearson, Naucalpan (1992)

15. Marques de Sá, J.P.: Pattern Recoanition: Concepts, Methods and Applications. Springer, Oporto (2001)

16. Pratt, W.K.: Digital image processing. John Wiley \& Sons, Inc., New York (2001) 